\title{
Les Pueblos culturales de la Sierra Nevada de Santa Marta : volet social d'une stratégie de développement économique?
}

\section{Clélie Parriche}

\section{(2) OpenEdition}

Journals

Édition électronique

URL : https://journals.openedition.org/jsa/11990

DOI : 10.4000/jsa.11990

ISSN : $1957-7842$

Éditeur

Société des américanistes

Édition imprimée

Date de publication : 20 décembre 2011

Pagination : 369-381

ISSN : 0037-9174

Référence électronique

Clélie Parriche, "Les Pueblos culturales de la Sierra Nevada de Santa Marta : volet social d'une stratégie de développement économique? », Journal de la Société des américanistes [En ligne], 97-2 I 2011, mis en ligne le 22 janvier 2012, consulté le 06 septembre 2022. URL : http:// journals.openedition.org/jsa/11990; DOI : https://doi.org/10.4000/jsa.11990 


\section{COLOMBIE}

\section{LES PUEBLOS CULTURALES DE LA SIERRA NEVADA DE SANTA MARTA : VOLET SOCIAL D'UNE STRATÉGIE DE DÉVELOPPEMENT ÉCONOMIQUE?}




Depuis 2007, la région de la Sierra Nevada de Santa Marta (SNSM), au nord-est de la Colombie, est l'objet d'une vaste politique de récupération territoriale entreprise durant le premier mandat du président Álvaro Uribe Vélez (2002-2006) dans le cadre de sa Politique de Sécurité Démocratique. Vaste massif montagneux situé au bord des Caraïbes ${ }^{1}$, la Sierra Nevada constitue aujourd'hui une région stratégique pour le développement du pays, non seulement de par sa situation géographique - à proximité de l'océan Atlantique elle représente un vaste couloir de circulation depuis la frontière vénézuélienne, à l'est, jusqu'à l'Urabá, près de la frontière panaméenne, à l'ouest du pays -, mais également du point de vue de sa riche biodiversité et de ses innombrables ressources naturelles. Après la reconquête militaire de la zone, en 2004, une récupération dite " sociale » devait parvenir à intégrer cette région marginalisée au territoire national. L'objectif de cette politique était de rétablir la gouvernabilité des territoires en garantissant la présence et la légitimité de l'État dans la région, tout en tentant de restituer la confiance des citoyens à l'égard des institutions étatiques, via le renforcement des organes locaux de gouvernement et le déploiement des services sociaux ${ }^{2}$.

Cette politique s'est concrétisée par la mise en œuvre du programme Cordón ambiental y tradicional de la Sierra Nevada de Santa Marta (soit «Ceinture environnementale et traditionnelle de la Sierra Nevada de Santa Marta »), lancé en 2007 par le président Uribe. Ce programme consistait dans la construction d'une dizaine de villages indigènes autour du massif de la Sierra. Il avait pour objectif de fournir les services publics élémentaires de l'État aux populations indigènes de la région, tout en assurant la permanence culturelle de ces groupes et en veillant à la préservation de leur environnement et de leurs richesses naturelles. L'octroi préalable de nouvelles terres aux communautés ${ }^{3}$ pour la construction des villages répondait par ailleurs aux revendications formulées depuis de nombreuses années par les Indiens de la Sierra Nevada réclamant l'extension des resguardos ${ }^{4}$ existants et la reconnaissance de l'intégralité de leur territoire ancestral.

Le succès de ce programme auprès des communautés indigènes de la Sierra a amené l'État à étendre l'initiative : de nouveaux villages sont aujourd'hui en construction dans la région et le nouveau gouvernement étudie la possibilité de lancer un programme identique dans la municipalité de Mutatá, dans le département d'Antioquia, avec les communautés indigènes embera katío ${ }^{5}$. C'est que les promesses d'extension et de récupération des terres ancestrales qui accompagnent cette initiative constituent une réponse concrète aux revendications historiques de certaines communautés indiennes du pays qui luttent depuis des décennies pour récupérer leur territoire traditionnel. Dans la Sierra Nevada, la construction des villages culturels a en effet permis aux quatre communautés indiennes de se rapprocher de la línea negra, ligne imaginaire qui délimite leur territoire ancestral ${ }^{6}$. Les autorités gouvernementales se 
félicitent, quant à elles, du résultat en terme de développement. Disputée par les paramilitaires et les guérillas au cours des années 1990, marginalisée vis-à-vis du territoire national pendant des décennies, la Sierra Nevada est aujourd'hui une région sécurisée et son potentiel économique attire chaque jour de nouveaux investisseurs.

Aussi, derrière les ambitions socio-culturelles et environnementales affichées au moment du lancement du programme, le gouvernement colombien laisse-t-il aujourd'hui s'exprimer d'autres «appétits», qui révèlent les ambitions économiques de l'État à l'égard de la région. Cette zone occupe une position trop stratégique du point de vue du développement économique pour que la majeure partie de son territoire soit confiée aux Indiens. Depuis l'arrivée du président Juan Manuel Santos à la tête du gouvernement, les annonces se multiplient pour fomenter le potentiel touristique et agro-industriel de la région. Face à cette ouverture annoncée du développement, certains villages indigènes construits dans des zones particulièrement stratégiques voient aujourd'hui leur permanence menacée par les intérêts économiques qui s'affrontent pour le contrôle du territoire. Les Kankuamo sont une des populations les plus durement touchées par ces nouveaux conflits.

\section{La Sierra Nevada de Santa Marta, zone d'intervention Prioritaire}

Théâtre d'affrontements sanglants entre guérilleros, narcotrafiquants et paramilitaires dans les années 1990, la Sierra Nevada a été une des zones prioritaires d'intervention dans le cadre de la mise en œuvre de la Politique de Sécurité Démocratique du président Álvaro Uribe Vélez. Dès 2004, elle a fait l'objet d'une vaste opération de contrôle militaire qui a abouti à la démobilisation des groupes d'autodéfense et au démantèlement des mafias et groupes insurgés qui sévissaient dans la région. Fort de ce succès militaire et désireux de parvenir à une récupération totale du territoire, l'État a ensuite développé une politique sociale particulière à l'égard des communautés indigènes présentes dans le massif à travers l'exécution du programme du Cordón ambiental y tradicional de la Sierra Nevada de Santa Marta. Cette politique de récupération dite «sociale » du territoire s'est traduite par la construction d'une dizaine de villages indigènes dans les bassins des principaux fleuves qui parcourent la Sierra, destinés à accueillir les populations indigènes réfugiées sur les hauteurs du massif et confrontées à des problèmes majeurs de nutrition et de santé.

L'objectif de cette initiative était double. Dans un premier temps, elle visait à freiner la colonisation de la Sierra par les groupes armés illégaux, en assurant la présence militaire de l'État dans les bassins des grands fleuves qui sont autant de voies stratégiques de colonisation. La ceinture formée par les villages construits au pied de la Sierra devait constituer une sorte de $«$ talenquère $»{ }^{7}$ le 
long de laquelle l'armée colombienne pourrait circuler afin d'éloigner les éventuels hors-la-loi qui voudraient réinvestir la zone. Dans un second temps, le programme constituait un moyen pour déployer les services publics de l'État sur une des zones périphériques du pays, où la présence étatique avait été jusque-là très fragile, voire inexistante. Construits selon les traditions indigènes des quatre groupes locaux, les pueblos talenqueras, comme ils furent initialement dénommés, ont donc tous été dotés d'infrastructures occidentales destinées à fournir les principaux services publics de l'État en matière de santé, d'éducation et d'alimentation. Cette stratégie, mise en œuvre dans d'autres zones « rouges ${ }^{8}$ du pays, représentait une étape supplémentaire vers la territorialisation totale de l'action publique ${ }^{9}$.

Un troisième objectif a ensuite été assigné à cette politique : la protection environnementale de la région et la préservation des populations traditionnelles qui l'habitent. En effet, en permettant aux habitants des zones hautes de descendre occuper de nouveaux espaces et de libérer les territoires où s'exerçait une pression trop forte sur les ressources naturelles, la construction de ces villages au pied du massif de la Sierra devait non seulement permettre la préservation et la régénération des zones endommagées, mais aussi garantir la survie des groupes traditionnels de plus en plus touchés par la pauvreté et la sous-alimentation. D'où le nom de « Ceinture environnementale et traditionnelle de la Sierra Nevada de Santa Marta » attribué au programme destiné à concrétiser cette politique de récupération sociale du territoire. L'inclusion de ce volet environnemental et culturel a par ailleurs amené les autorités gouvernementales à modifier l'adjectif originellement utilisé pour qualifier les villages indigènes : l'épithète talenquera a été remplacée par le mot cultural.

Placée sous le leadership du groupe Arhuaco à l'origine de l'initiative, la mise en œuvre de ce programme n'a pas bénéficié de la même manière aux quatre groupes. Les Kankuano sont ceux qui ont été le moins favorisés : ils ont obtenu la construction d'un seul village, Magugueka, dans le bassin du fleuve Guatapurí, dans la municipalité de Valledupar (département du César), sur la face sud-est de la Sierra. Si le facteur politique semble expliquer, dans un premier temps, le succès des communautés arhuaco et kogi ${ }^{10}$ au cours des négociations avec le gouvernement, un autre facteur d'ordre économique éclaire la faiblesse de la position des Kankuano. Tout comme les autres projets, la construction du village de Magugueka (« le lieu pour éduquer » en kankuamo) devait venir soutenir la stratégie de renaissance culturelle entreprise depuis plusieurs années par les Kankuano, la reconnaissance progressive de leur territoire ancestral devant être le gage de la survie et du renforcement de leur communauté. Néanmoins, à la différence des autres constructions, celle-ci a été réalisée sur une zone devenue stratégique pour le développement économique du département du César. 


\section{LE BASSIN DU FLEUVE GUATAPURí : UN TERRITOIRE HISTORIQUEMENT CONFLICTUEL}

Magugueka est situé dans le bassin d'un des fleuves les plus importants de la Sierra Nevada, le fleuve Guatapurí, sur le versant est de la montagne. Cette zone, où confluent aujourd'hui trois des quatre peuples originels de la région (Kogi, Arhuaco et Kankuamo), a été un point de rencontre et d'échanges historique entre le monde indigène et les différentes sociétés qui ont colonisé la Sierra depuis 250 ans. Il a aussi été le théâtre de nombreux conflits et affrontements tout au long des $\mathrm{XIX}^{\mathrm{e}}$ et $\mathrm{XX}^{\mathrm{e}}$ siècles entre des populations aux intérêts profondément divergents. Au cours de ces oppositions, les rapports de forces ont souvent été très inégaux et largement défavorables aux communautés indiennes habitant le bassin du fleuve.

Sur le versant est du massif, le fleuve Guatapurí définit la frontière entre les territoires respectifs des quatre groupes. Selon leur histoire traditionnelle, chaque peuple indigène de la Sierra possède une " aire ancestrale spécifique » qui constitue la base de sa culture, de sa survie et dont il a la responsabilité spirituelle ${ }^{11}$. À la fin du XIX ${ }^{\mathrm{e}}$ siècle, la présence accrue des missionnaires catholiques à Atanquez, centre du monde traditionnel des Kankuamo, a été à l'origine d'un lent processus d'abandon de leur culture ancestrale. Les membres de ce groupe se sont alors trouvés dans l'incapacité d'assurer la préservation spirituelle de leur territoire ${ }^{12}$. Aussi, face à la perte d'autorité de leurs mamos (les autorités spirituelles de la communauté), des accords interethniques ont été passés afin de confier cette responsabilité à des familles kogi qui, poussées par la multiplication des fronts de colonisation sur le versant nord de la Sierra Nevada où se trouvait leur territoire ancestral, étaient venues se réfugier sur le versant sud-est du massif, dans le bassin du fleuve Guatapurí. Ces familles furent chargées de la préservation des sites sacrés et des connaissances des Kankuamo sur leur territoire, jusqu'à ce que ces derniers puissent de nouveau « en faire usage dans de meilleures conditions culturelles et spirituelles » (Aja Eslava 2010, p. 164).

Néanmoins, les Kankuamo auront d'autant plus de difficultés à revaloriser leurs savoirs et leurs coutumes que, après l'installation des colons évangélisateurs ${ }^{13}$, la vallée du bassin du fleuve Guatapurí est devenue l'objet de convoitises économiques des éleveurs de la Sierra. Depuis la fin du XVIII ${ }^{\mathrm{e}}$ siècle, l'élevage avait été l'activité principale des familles aisées de la province de Santa Marta, qui avait juridiction dans la partie orientale de la région Caraïbe. Cette activité avait été largement adoptée dans la Sierra et avait progressivement remplacé l'agriculture. La propriété de la terre s'était transformée à mesure que les grandes familles d'éleveurs élargissaient leurs domaines. Les relations interethniques s'étaient aussi modifiées, pour aboutir à une inégalité de fait entre les sociétés indigènes et non indigènes ${ }^{14}$. La réforme agraire apporta un coup final à la propriété collective traditionnelle des Indiens de la région. 
Devant la perte de contrôle de leur territoire, certaines communautés commencèrent à s'organiser. C'est le cas des Arhuaco qui, après un long processus de rassemblement des autorités des différentes communautés occupant le massif, allèrent interpeller le gouvernement colombien pour faire reconnaître leurs droits sur leurs terres ancestrales. Au début des années 1970, alors qu'émergent dans le pays les premiers mouvements indigènes, l'État colombien crée la reserva arhuaca et reconnaît l'existence symbolique de la línea negra comme limite imaginaire du territoire ancestral des quatre communautés de la Sierra Nevada. En 1982, la réserve, dont la superficie a été agrandie, adopte le statut de resguardo ${ }^{15}$. Ces créations vont être décisives pour la lutte des populations indigènes. Le rapport de force changera peu à peu : « de sociétés dominées, privées de droits, discriminées et sans capacité de décision sur leur territoire, [les communautés indigènes] vont être perçues comme des entités autonomes, véritablement propriétaires de leur territoire, détentrices d'une culture ancestrale et dotées d'autorités propres capables de gouverner leur peuple » (Aja Eslava 2010, p. 165 ; traduction en français de l'auteur, C. P.).

Ces changements, qui ouvrent la voie à la reconnaissance de nouveaux droits particuliers et collectifs des communautés indigènes du pays ${ }^{16}$, concernent d'abord les communautés arhuaco de la Sierra, puis celles des Kogi et des Wiwa qui disposent déjà d'organes de représentation ${ }^{17}$. Les Kankuamo, qui ont le plus souffert des mouvements successifs de colonisation dans la région, mettront plus de temps à faire reconnaître leurs droits par l'État colombien. Leur manque de légitimité en tant que "peuple indien originel » ralentira considérablement leur participation aux luttes déjà menées par les autres groupes. Ce ne fut qu'au début des années 1980 qu'ils entreprirent de lutter collectivement pour faire valoir leurs droits. Le processus de « récupération culturelle », amorcé au début de la décennie, a marqué leur « renaissance» en tant que peuple indien et a constitué le premier pas vers une prise de position nouvelle en tant qu'interlocuteurs incontournables du gouvernement.

Dans la dernière décennie du $\mathrm{xx}^{\mathrm{e}}$ siècle, les Kankuamo vont néanmoins se trouver confrontés à un autre type de colonisation, plus violent et plus menaçant pour leur survie. Au début des années 1990, le territoire du bassin du fleuve Guatapurí devient l'épicentre du conflit armé dans la région. Tout au long des quinze années durant lesquelles se poursuivront les affrontements, cette zone sera, successivement, le centre d'opération des divers fronts guérilleros, comme les Fuerzas armadas revolucionarias de Colombia (FARC) et l'Ejército de Liberación Nacional (ELN), repliés dans la région, le terrain d'action des Autodefensas unidas de Colombia (AUC) et une zone stratégique pour l'exportation des produits de la drogue. Les zones basses et moyennes du bassin deviendront alors les théâtres d'intenses combats entre acteurs armés pour le contrôle de ce territoire ${ }^{18}$.

Durant cette période, quelque quatre cents Indiens seront assassinés dans la juridiction du bassin et de nombreuses familles seront déplacées (Aja Eslava 
2010, p. 166). La communauté kankuamo sera durement touchée par les affrontements de ces groupes armés. Assassinats, déplacements, enrôlement forcé des jeunes, stigmatisation de la population mettront gravement en danger la survie physique et culturelle du peuple kankuamo. Devant le silence et l'inertie des autorités nationales à l'égard des exactions répétées des groupes armés à leur encontre, les Kankuamo seront alors forcés de chercher l'appui d'instances et d'organismes internationaux. Le travail de récupération culturelle entrepris par les autorités spirituelles et certains leaders de la communauté sera accompagné d'un difficile processus de dénonciation et de pétition afin de freiner l'extermination systématique de ses membres.

Si l'octroi de mesures provisoires par la Cour Interaméricaine des Droits de l'Homme (CIDH) de l'Organisation des États américains (OEA) au bénéfice des Kankuamo ${ }^{19}$, puis la constitution du resguardo indígena kankuamo ${ }^{20}$ au début des années 2000, ont récompensé leur lutte et consolidé leur pouvoir politique dans la région, il reste beaucoup à faire pour que soit garantie leur pérennité physique et culturelle. Le conflit armé a provoqué une rupture profonde dans la dynamique de mémoire enclenchée après l'expulsion des communautés catholiques de la région. Aujourd'hui, encore affaiblie et fortement divisée, la communauté kankuamo souffre d'autant plus des pressions exercées par certains acteurs intervenant dans la région qu'elle ne parvient pas à rallier l'ensemble de ses membres autour d'un même projet collectif.

\section{Le VILlage de MagugueKa AU CENTRE deS lutTes TerRitoriales}

Malgré la fin du conflit, les luttes n'ont pas cessé pour le contrôle des terres ancestrales des Kankuamo. Aujourd'hui plus que jamais, ces terres constituent un espace stratégique dans la région. Ces dernières années, les manœuvres spéculatives des grands propriétaires terriens ont, comme nous l'avons dit, pris le relais des affrontements armés. Plusieurs projets participent actuellement à la valorisation spéculative du territoire traditionnel des Kankuamo : la construction d'un réservoir multifonctionnel sur le fleuve Guatapurí destiné à alimenter en eau la municipalité de Valledupar et certaines communes voisines, celle d'une route de conteneurs pour relier le Venezuela voisin, sur une voie stratégique qui constitue l'unique sortie vers la mer Caraïbe à l'est du massif, enfin celle d'un aéroport au nord du département. Outre ces trois projets, les propriétaires locaux semblent aussi compter sur le développement touristique de la région. Depuis la démobilisation des groupes armés, les autorités locales multiplient en effet les initiatives destinées à promouvoir la découverte touristique du patrimoine culturel et écologique de la Sierra Nevada.

Aussi, dans son combat pour la récupération de son territoire traditionnel, la communauté kankuamo doit-elle faire face non seulement à l'opposition des 
grands propriétaires terriens qui refusent de vendre leurs terres, mais également aux arguments économiques avancés par les autorités locales intéressées au développement de la région. Parfois alliés dans leurs luttes, ces deux acteurs sont parvenus à limiter l'extension du nouveau village culturel de Magugueka, dont la configuration actuelle ne permet pas de développer les activités d'autosubsistance nécessaires à la vie quotidienne de ses habitants ${ }^{21}$. Ils continuent aujourd'hui à faire pression sur les populations du village pour qu'aucune initiative nouvelle ne soit prise par les habitants. Certains évoquent les menaces de «forces obscures » ${ }^{22}$ qui viennent rôder le soir autour du village, afin de les intimider, ces mêmes forces qui ont terrorisé les populations jusqu'au début des années 2000 et qui ont été à l'origine des nombreuses exactions commises à l'encontre de la communauté.

À cette pression et à ces menaces, s'ajoutent des divisions internes au sein de la communauté. L’Organización Indígena Kankuama (OIK), créée en 1993, est censée représenter les intérêts des douze communautés qui vivent dans le resguardo kankuamo. Néanmoins, certains secteurs de la population ont toujours refusé les directives de l'organisation. Aujourd'hui, l'Organización Indígena Atanquez Libre, qui a adopté l'épithète "indigène » depuis peu pour mieux concurrencer l'OIK ${ }^{23}$, s'oppose frontalement aux actions de cette dernière. Ces deux organisations ne partagent pas la même vision à l'égard des actions à mener dans le resguardo et nouent des alliances avec des acteurs aux intérêts profondément divergents. Actuellement, elles s'opposent notamment sur la politique économique à mener dans la réserve : alors que les membres de l'OIK promeuvent la mise en œuvre d'un programme gouvernemental de développement dans lequel $70 \%$ du territoire serait dédié à la conservation et $30 \%$ à des activités d'autosubsistance, l'organisation Atanquez Libre défend le développement d'une activité agricole qui couvre la totalité du resguardo, ainsi que la commercialisation de l'intégralité des produits tirés de cette activité. Tandis que l'OIK est soutenue par des ONG environnementales locales et nationales, Atanquez Libre s'est rapprochée des autorités municipales ${ }^{24}$ et s'est opposée, aux côtés des exécutifs locaux, au programme de la « Ceinture environnementale et traditionnelle de la SNSM ».

Toutefois, malgré ces divisions internes et ces divergences d'intérêts, la construction du village culturel de Magugueka a pu être menée à bien. Achevé en 2010, il est aujourd'hui opérationnel. Les premières familles ont commencé à habiter les maisons traditionnelles, un programme d'éducation biculturelle est en cours d'élaboration et le centre de santé fonctionne. Les représentants arhuaco, qui ont promu le projet, célèbrent aujourd'hui le nouveau rapport de force établi grâce à ce programme et les avancées réalisées dans leur lutte pour la récupération de leurs terres ancestrales. Selon eux, il a permis une réelle implication des communautés indigènes de la Sierra Nevada qui peuvent aujourd'hui négocier d'égal à égal avec le pouvoir central et les autres acteurs intéressés par leur 
territoire. Toutefois, dans la cas de Magugueka, la pression des grands propriétaires voisins, les menaces permanentes de «forces obscures», l'absence d'entente avec les autorités politiques locales, quant à la nécessité d'élargir la zone du village pour mettre en place des activités d'autosubsistance, pèsent lourdement sur sa mise en fonctionnement, en même temps qu'elles compromettent l'espoir de renouveler une telle expérience avec la construction d'un autre village.

\section{UN ÉQUILIBRE PROVISOIRE DES FORCES}

Au cours des 150 dernières années, le groupe kankuamo a souffert de différents types d'intrusions et de menaces: la présence des missions catholiques et évangéliques ; l'occupation des terres basses du territoire par des colons paysans et des grands propriétaires terriens pratiquant l'agriculture et l'élevage intensif qui se sont progressivement insérés dans la vie des communautés et ont menacé le maintien de leurs valeurs culturelles ; la présence d'acteurs armés et de groupes économiques qui, parfois avec la complicité des autorités locales, ont mis en danger leur intégrité physique. Face à ces dangers, nombreux sont les Kankuamo qui ont été amenés à nier ou à occulter leurs origines et à abandonner les pratiques traditionnelles qui les liaient aux autres groupes de la région. Si les Kankuamo ont pu participer au projet de la «Ceinture environnementale et traditionnelle de la SNSM », c'est grâce à l'initiative de certains leaders qui continuèrent à consulter les autorités traditionnelles de leurs communautés, ainsi qu'au travail d'enseignants qui, avec le soutien d'organisations locales et internationales, tentent aujourd'hui de revitaliser auprès des jeunes une culture restée longtemps invisible.

Toutefois, moins que la démonstration du poids politique acquis par les Indiens dans leur lutte pour la consolidation et l'extension de leur territoire, la construction du village culturel de Magugueka semble surtout révéler les limites de la générosité de l'État colombien dans sa politique de soutien au processus de récupération territoriale des communautés indigènes de la Sierra Nevada. En effet, compte tenu des pressions exercées sur la communauté kankuamo au moment de la construction du village et au regard des politiques actuellement menées en dehors du cadre du programme de la « Ceinture environnementale et traditionnelle de la SNSM », c'est un bilan réservé que l'on doit tirer de l'intervention actuelle du gouvernement central dans la région et de la politique de participation qui est menée à l'égard des communautés indigènes. C'est en fait un bilan en demi-teinte qui relativise la marge de manœuvre des communautés indigènes et qui confirme la puissance des intérêts économiques qui parviennent à influencer de manière décisive l'action du gouvernement colombien.

En effet, si, dans un premier temps, le programme de la "Ceinture environnementale et traditionnelle de la SNSM » a semblé constituer une promesse 
sérieuse d'extension des réserves indigènes actuelles, validant le pouvoir politique acquis par les communautés, la multiplication des projets de développement économique envisagés dans la région fait aujourd'hui craindre une instrumentalisation des quatre communautés par le gouvernement central, qui souhaite mettre définitivement la Sierra Nevada sous contrôle, afin de permettre son ouverture au tourisme et à l'exploitation de ses ressources naturelles. L'État ne renonce pas aux richesses économiques de ce territoire classé «Réserve de l'homme et de la biosphère » par l'Unesco, comme en témoigne le projet de téléphérique vers le site archéologique, et désormais touristique, de Ciudad Pérdida, dans le département du Magdalena, à l'ouest du massif, ou celui de la construction d'un barrage hydraulique sur le territoire traditionnel des Indiens wiwa et kogi dans le département de La Guajira, au nord-est.

Ce sont surtout les ressources naturelles qui suscitent l'intérêt du gouvernement et celui des investisseurs. Outre la présence d'importantes ressources hydrauliques, la région dispose également d'un riche potentiel minier. Ces dernières années, l'exploitation du charbon a suscité un intérêt accru pour la région, et le département du César est devenu l'un des principaux producteurs et exportateurs du pays. Si l'exploitation minière fait naître des inquiétudes au sein de la population et parmi les autorités gouvernementales locales quant à ses conséquences néfastes sur l'environnement, il n'en est pas de même pour la question de l'exploitation des ressources hydrauliques. Confrontés aux réclamations des habitants régulièrement affectés par les coupures d'eau, mais aussi aux problèmes de sécheresse qui compromettent les activités agricoles du département, les élus locaux du César ont entrepris de relancer les négociations avec les communautés indigènes avant d'entreprendre les démarches pour l'obtention d'une licence d'exploitation du fleuve Guatapurí. Le maire et le gouverneur font cause commune pour promouvoir le projet auprès du gouvernement central. Ils défendent l'urgence de construire un réservoir permettant d'approvisionner la municipalité de Valledupar et d'autres communes voisines qui sont de plus en plus confrontées aux problèmes de pénurie d'eau.

Dans cette nouvelle configuration de promotion économico-touristique de la région, la communauté kankuamo risque d'être à nouveau la moins bien placée pour négocier, son territoire ancestral prenant chaque jour un peu plus de valeur. Les résidences secondaires de tourisme se multiplient aux abords de Magugueka, alors que, sous l'impulsion du ministère du Commerce, les autorités départementales viennent d'annoncer un programme conjoint entre les différentes municipalités du César pour promouvoir le potentiel touristique de la région. Lors d'une visite récente dans la municipalité de Valledupar, le ministre du Commerce a insisté sur la nécessité de faire, au niveau national et international, la promotion du potentiel touristique de la région : son folklore, sa gastronomie, son artisanat et ses activités d'écotourisme devant devenir autant d'éléments attractifs pour les voyageurs colombiens et étrangers ${ }^{25}$. Une quinzaine de jours après cette visite, le 
maire de Valledupar plaidait, à son tour, pour le développement culturel et touristique de la région à l'Expo Colombia Internacional, dans la ville de Miami ${ }^{26}$. Avec de telles initiatives, le prix de la terre risque de s'envoler à nouveau dans la Sierra Nevada et, avec lui, l'espoir des communautés indigènes de récupérer une nouvelle partie de leur territoire traditionnel.

Clélie PARRICHE

Paris Sorbonne Nouvelle/IHEAL

\section{NOTES}

1. La SNSM représente environ $17000 \mathrm{~km}^{2}$. Sur cette vaste superficie, cohabite une population d'une grande diversité et se juxtapose une multiplicité de juridictions, d'ordre administratif, politique et environnemental. Outre les quatre communautés indigènes, Kogi, Arhuaco, Wiwa et Kankuamo, peuples originels de la SNSM, on relève, dans les zones basses du massif, la présence de familles de paysans et celle de grands propriétaires terriens qui occupent les vallées où ils pratiquent l'élevage et la culture d'huile de palme. On note aussi la présence d'entreprises d'exploitation minière et de tourisme, ainsi que celle d'organisations environnementales nationales et internationales. Trois départements, ceux du Magdalena, du César et de La Guajira, et dix-huit municipalités possèdent juridiction dans la SNSM. Est aussi présente l'Unidad Administrativa Especial de Parques Naturales Nacionales (UAEPNN), organisme dépendant du ministère de l'Environnement, qui gère les deux Parcs naturels de la région et dont la juridiction se superpose très souvent à celle des trois réserves indigènes. Enfin, à certaines de ces juridictions, se superposent parfois des zones particulières d'exploitation, concédées à des acteurs privés impliqués dans le tourisme ou dans l'extraction minière.

2. Por la recuperación social del territorio, Centro de Coordinación Integral de Acción (CCAI), junio 2006.

3. Les Kogi, Arhuaco, Wiwa et Kankuamo sont les quatre groupes qui peuplent la SNSM.

4. La figure juridique du resguardo, institution d'origine coloniale, reconnaît un territoire collectif inaliénable appartenant aux membres d'une communauté ou parcialidad indigène. Trois resguardos ont été créés dans la région depuis les années 1970 : le resguardo arhuaco, sur le versant sud du massif, le resguardo arhuaco-wiwa-kogi, sur le côté nord de la Sierra, et le resguardo kankuamo, sur la face sud-orientale de la montagne.

5. Information non publiée, obtenue lors d'un entretien auprès de Carolina Alvaro, collaboratrice de la Agencia Presidencial para la Acción Social, entité gouvernementale chargée de l'élaboration et de la mise en œuvre du programme, 25 novembre 2010, Bogotá, Colombie.

6. La résolution 000002 du 4 janvier 1973 reconnaît les différents sitios sagrados (« sites sacrés ») des quatre communautés kogi, wiwa, arhuaco et kankuamo. Parmi ces sites sacrés, ceux qui se trouvent en aval des fleuves forment une ligne imaginaire qui délimite leur territoire ancestral, c'est la fameuse linea negra. La résolution 837 du 28 août 1995 reconnaît officiellement l'existence et le concept de la línea negra [voir l'ouvrage réalisé par Organización Indígena Kankuama (OIK), Modelo participativo de Ordenamiento del resguardo indígena kankuamo, Fundación E-Korúa, coll. "Kampanäkê », Valledupar, Ceqar, 2006]. Lorena Aja Eslava définit la línea negra à partir du concept kogi sénénulang qui exprime l'idée d'intégralité et qui renvoie au « territorio ancestral definido por las relaciones y los manejos ancestrales, que los pueblos indígenas han hecho del hábitat, y dado su importancia cultural y de supervivencia material, se convierte en la médula de la política de los pueblos indígenas a la hora de relacionarse con el estado nacional » (Aja Eslava 2010, p. 177).

7. Le mot « talenquère » (talenquera en espagnol) est utilisé, dans la tauromachie, pour désigner la barrière qui, au sein d'une arène, est située autour de la piste et derrière laquelle les participants peuvent se protéger. 
8. Ces zones dites « rouges" se caractérisent par un état de violence qui affecte directement le développement des régions et qui limite le bien-être de leurs habitants, ainsi que par une faible présence étatique, au niveau régional, ce qui empêche de garantir des conditions de vie satisfaisantes à tous les Colombiens et qui rend difficile la résolution de conflits, l'application efficace de la loi et le monopole de l'usage de la violence légitime (García et Sarmiento 2002, p. 2).

9. Jolly (2008) recourt à la notion de « territorialité partielle » pour exprimer le fait que l'autorité de l'État colombien et sa capacité à répondre aux demandes des citoyens ne couvrent pas la totalité du territoire. La territorialisation de l'action publique est dite « partielle ».

10. Ces deux groupes bénéficient d'une certaine aura internationale et constituent des acteurs politiques de poids dans la région. Ils ont obtenu, respectivement, la construction de cinq et quatre villages. Les Wiwa, quant à eux, étaient d'abord réfractaires au programme, puis ont fini par solliciter la construction d'un village.

11. "Estas delimitaciones corresponden a las tareas tradicionales, las que tienen que ver con el mundo espiritual. Son los límites que la Madre espiritual adjudicó a cada pueblo para que lo cuidara por medio de trabajos espirituales, tales como los rituales de pagamento. Es decir que estos límites no implican necesariamente ubicación, ocupación o propiedad sobre la tierra por parte del pueblo indígena asignado. Lo que determinan son las responsabilidades que tiene cada pueblo y sus respectivas autoridades para con el cuidado y conservación espiritual del territorio ancestralmente adjudicado » (Aja Eslava 2010, p. 163).

12. Leur " aire ancestrale traditionnelle », qui comprend un espace allant du bassin du Donachwí jusqu'à celui du fleuve Badillo, est celle qui couvre la plus grande partie du fleuve Guatapurí.

13. La colonisation des territoires indigènes de la Sierra par des missions catholiques fut constante à partir de la fin $\mathrm{du} \mathrm{XVIII}^{\mathrm{e}}$ siècle, mais elle se renforça au début du $\mathrm{Xx}^{\mathrm{e}}$ siècle avec l'arrivée de missionnaires capucins à Nabusímake, centre du monde traditionnel arhuaco, qui provoqua une colonisation massive. La politique évangélisatrice de ces missionnaires força les Arhuaco à migrer vers d'autres terres, notamment en direction du bassin Guatapurí, où les Kogi étaient déjà venus se réfugier.

14. "Quienes antes eran dueños efectivos del territorio (los indigenas) pasan por ser ocupantes, previamente autorizados por los dueños y generándose unas relaciones paternalistas y de compadrazgo que facilitan esta ocupación del territorio y el disfrute de las ganancias que la actividad ganadera genera » (Aja Eslava 2010, p. 165). Progressivement, plusieurs communautés indigènes adoptèrent l'élevage et l'introduisirent dans les zones hautes de la Sierra, où les élites locales n'ont pas le contrôle des terres. Cette nouvelle activité sera par ailleurs à l'origine de l'introduction progressive de la monnaie. Son usage se consolida avec l'adoption d'autres activités productives, comme le café, la commercialisation de l'avocat ou la vente de mochilas traditionnelles.

15. Les articles 329 et 330 de la Constitution colombienne de 1991 considèrent les resguardos comme des entités territoriales au même titre que les districts, les municipalités et les départements. En tant que tels, ils disposent de l'autonomie politique et d'une participation dans les revenus courants de la nation.

16. La Constitution de 1991 va reconnaître des droits particuliers et collectifs aux minorités indigènes du pays, parmi lesquels le droit d'administrer leurs territoires et leur population selon leurs us et coutumes et avec des autorités qui leur sont propres.

17. La Confederación indígena tayrona (CIT), organisation du peuple arhuaco, est créée dans les années 1970; l'Organización gonawindúa tayrona (OGT) des Kogi, est fondée en 1987; l'Organización wiwa yugumaiun bunkwanarrwa tayrona (OWYBT) apparaît plus tard, au début des années 1990.

18. Au cours des années 1990, le développement du narcotrafic dans la SNSM accrût considérablement les violences dans cette région.

19. Dans la première ordonnance de mesures conservatoires du 5 juillet 2004, la Cour Interaméricaine des Droits de l'Homme demandait, en premier lieu, à l'État colombien de protéger la vie et l'intégrité personnelle de tous les membres des communautés qui forment le peuple autochtone kankuamo. Elle ordonnait également à l'État de réaliser des enquêtes sur ce qui motivait l'adoption de ces mesures provisoires, de garantir le droit à la libre circulation des Kankuamo, de faire participer 
la population aux décisions et à la planification des mesures qui seraient prises et de la tenir régulièrement informée.

20. Le resguardo kankuamo a été créé en 2003. Il se trouve dans la municipalité de Valledupar, département du César, sur le versant sud-est du massif. Il représente 24000 hectares et comprend douze communautés dans les corregimientos de Atanquez, La Mina, Guatapurí, Chemesquemena, Los Haticos y Río Seco, les Veredas de Ramalito, Rancho de la Goya, El Mojao, El Ponton Murillo et Las Flores. La population kankuamo comprend environ 13000 individus, dont $65 \%$ vivent au sein du resguardo et les $35 \%$ restants correspondant à la population déplacée, dans la ville de Valledupar, capitale du César, ou dans d'autres zones du pays [voir l'ouvrage réalisé sous les auspices de la United States Agency International Development (USAID), Hoja de cruz, memoria histórica del conflicto armado en el Pueblo indigena Kankuamo, Ediciones KUINO, 2006].

21. C'est un des problèmes centraux majeurs des communautés kankuamo habitant le resguardo. Seulement $3 \%$ du resguardo kankuamo serait apte à la production, la majorité des terres ne pouvant être destinée qu'à un usage forestier. À cela s'ajoute des niveaux d'érosion considérable, de sécheresse et de fragmentation, qui posent le problème critique des activités productives dans la réserve : voir l'ouvrage Hoja de cruz..., cité dans la note 20.

22. Expression tirée d'un entretien réalisé en novembre 2011 auprès du trésorier de l'Organización Indígena Kankuama (OIK), José Damian Maestre Villazón, qui a mené les négociations avec le gouvernement au moment de la construction du village de Magugueka.

23. D’abord dénommée Comité Civil, puis Organización Atanquez.

24. La mairie de Valledupar a mis des bureaux à la disposition de l'organisation au sein du bâtiment municipal.

25. Voir « Mincomercio planteó explotar a la Sierra Nevada como destino turístico regional », El Pilón, 14 mars 2011 [en ligne : http://www.elpilon.com.co/inicio/mincomercio-planteo-explotar-ala-sierra-nevada-como-destino-turistico-regional/, consulté le 19/03/2011].

26. Voir « Alcalde de Valledupar emprende promoción turística de la ciudad en Miami », El Pilón, 7 avril 2011 [en ligne : http://www.elpilon.com.co/inicio/alcalde-de-valledupar-emprende-promocionturistica-de-la-ciudad-en-miami/, consulté le 09/04/2011].

\title{
RÉFÉRENCES CITÉES
}

\author{
Aja Eslava Lorena \\ 2010 Agua, territorio y poder : representaciones, significados, usos y manejos del \\ agua en la Sierra Nevada de Santa Marta. Estudio de caso, maestria en \\ estudios del Caribe, Universidad nacional de Colombia/Instituto de estudios \\ caribeños San Andrés. \\ García Arturo et Alfredo Sarmiento (éd.) \\ 2002 Programas regionales de desarrollo y paz : casos de capital social y desarrollo \\ institucional, Fundación Ideas Para la Paz/PNUD, Bogotá. \\ JOLLY Jean-François \\ 2008 Régir le territoire et gouverner les territoires. Décentralisation et territoriali- \\ sation des politiques publiques en Colombie, L'Harmattan, Paris.
}

